\title{
Boundary Layer Transition Flight Experiment Overview and In-Situ Measurements
}

\author{
Brian P. Anderson ${ }^{1}$, Charles H. Campbell ${ }^{2}$, Luis A. Saucedo ${ }^{3}$ \\ NASA Johnson Space Center, Houston, TX \\ Gerald R. Kinder ${ }^{4}$ \\ The Boeing Company, Huntington Beach, CA \\ Karen T. Berger ${ }^{5}$ \\ NASA Langley Research Center, Hampton, VA
}

In support of the Boundary Layer Transition Flight Experiment (BLT FE) Project, a manufactured protuberance tile was installed on the port wing of Space Shuttle Orbiter Discovery for the flights of STS-119 and STS-128. Additional instrumentation was also installed in order to obtain more spatially resolved measurements downstream of the protuberance. This paper provides an overview of the BLT FE Project, including the project history, organizations involved, and motivations for the flight experiment.

Significant efforts were made to place the protuberance at an appropriate location on the Orbiter and to design the protuberance to withstand the expected environments. Efforts were also extended to understand the as-fabricated shape of the protuberance and the thermal protection system tile configuration surrounding the protuberance. A high-level overview of the in-situ flight data is presented, along with a summary of the comparisons between pre- and post-flight analysis predictions and flight data. Comparisons show that predictions for boundary layer transition onset time closely match the flight data, while predicted temperatures were significantly higher than observed flight temperatures.

$\begin{array}{ll}\text { Acronyms } & \\ \text { BET } & \text { Best Estimated Trajectory } \\ \text { BLT } & \text { Boundary Layer Transition } \\ \text { BRI } & \text { Boeing Replacement Insulation } \\ \text { BLT FE } & \text { Boundary Layer Transition Flight Experiment } \\ \text { CDR } & \text { Critical Design Review } \\ \text { CFD } & \text { Computational Fluid Dynamics } \\ \text { DAT } & \text { Damage Assessment Team } \\ \text { DTO } & \text { Detailed Test Objective } \\ \text { EI } & \text { Entry Interface } \\ \text { EVA } & \text { Extravehicular Activity } \\ \text { FRSI } & \text { Felt Reusable Surface Insulation } \\ \text { HYTHIRM } & \text { Hypersonic Thermodynamic Infrared Measurements } \\ \text { ISS } & \text { International Space Station }\end{array}$

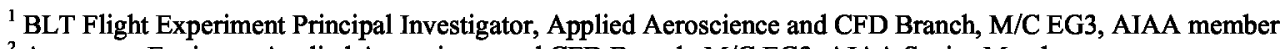

${ }^{2}$ Aerospace Engineer, Applied Aeroscience and CFD Branch, M/C EG3, AIAA Senior Member

${ }^{3}$ BLT Flight Experiment Project Manager, Orbiter Project Office, M/C MV6

${ }^{4}$ Senior Engineer/ Scientist, Integrated Defense Systems/Space Exploration, Flight Sciences/Aerodynamics

${ }^{5}$ Deputy BLT Flight Experiment Principal Investigator, Aerothermodynamics Branch, M/S 408A, AIAA Senior member 


$\begin{array}{ll}\text { JSC } & \text { Johnson Space Center } \\ \text { MADS } & \text { Modular Auxiliary Data System } \\ \text { MPLM } & \text { Multi-Purpose Logistics Module } \\ \text { OMS } & \text { Orbital Maneuvering System } \\ \text { PDR } & \text { Prelminary Design Review } \\ \text { PRCB } & \text { Program Requirements Control Board } \\ \text { RF } & \text { Radio Frequency } \\ \text { RID } & \text { Review Item Discrepancy } \\ \text { SE\&I } & \text { Systems Engineering \& Integration } \\ \text { SIP } & \text { Strain Isolation Pad } \\ \text { SRR } & \text { System Requirements Review } \\ \text { SSP } & \text { Space Shuttle Program } \\ \text { STS } & \text { Space Transportation System } \\ \text { TPS } & \text { Thermal Protection System } \\ \text { TUFI } & \text { Toughened Unipiece Fibrous Insulation }\end{array}$

Symbols
$B F_{C F D}$
$k$
$\mathrm{M}_{\mathrm{e}}$
$\operatorname{Re}_{\theta}$
$\dot{q}$
$\dot{q}_{X F 0002}$
$\sigma$

\section{Introduction}

The design and ultimate weight of any vehicle entering a planetary atmosphere is affected by the amount of thermal protection system (TPS) required for safe and successful entry. One of the drivers to the design and sizing of TPS is the time during the entry at which the boundary layer transitions from laminar to turbulent flow. The study of boundary layer transition (BLT) has been a significant effort for many decades. However, the specific physics-based mechanisms that cause BLT are poorly understood. This lack of understanding hinders designers in making accurate predictions of when the boundary layer will become turbulent and affects the sizing or the understanding of the robustness of a TPS. If the geometry of a vehicle is known, engineers can obtain ground-test data from wind tunnels to develop engineering correlations. [Ref Chuck's 2010 AIAA Paper, previous Berry papers] This method has been proven successful in several instances. [Ref Orbiter, MSL?, others?]. However, one of the major weaknesses in the groundbased correlation approach is a lack of understanding of the differences between the wind tunnel and flight environments and how those differences effect BLT. From a turbulence modeling perspective, very little data exists to verify turbulent heating prediction models at low Reynolds numbers and hypersonic conditions.

Following STS-107, the Damage Assessment Team (DAT) was established with a charter to assess the effects of ascent damage on safe Orbiter re-entry (Ref. Campbell 2006). A substantial analytical tool development activity was undertaken to characterize the 
aeroheating, thermal, and stress implications of damage on the Orbiter material temperatures, tile factors of safety, and structural margins. Aeroheating tools developed included the BLT Tool (Ref. Berry BLT vsn 1,2) and the Cavity Heating Tool (Ref Everhart, Boeing documents). In addition, the ability to rapidly produce Computational Fluid Dynamics (CFD) simulations of damage scenarios was also developed.

During STS-114 (Ref Berry San Francisco paper), an unprecedented repair spacewalk was performed to remove two protruding gap fillers because the risks associated with the uncertainties in early BLT and resulting heating effects were determined to be higher than the risks for spacewalk itself. As a result of these uncertainties with BLT and turbulent heating predictions, a flight test using the Space Shuttle Orbiter was proposed in November 2006 (Ref White Paper).

Several other flight experiments were also proposed including an experiment to obtain flight data in a tile cavity and to obtain detailed temperature measurements in the shockshock interaction region on the Orbiter wing leading edge. Initial requests to fund the flight-testing using supplemental funding to the SSP were declined. However, a flight experiment that was reduced in scope from original proposals was ultimately approved and funded by the SSP. Only the protuberance experiment was approved for funding. Early in the planning stages of the flight experiment, several proposals were presented, including a proposal to install experiment hardware on all three Orbiter vehicles. In the end, however, the protuberance tile and augmented instrumentation package was only installed on one Orbiter, OV-103 (Discovery). While many motivations exist (Ref. Chuck's 2008 AIAA Paper) to obtain flight test data of this kind, a strong desire to obtain data to improve modeling capabilities to increase potential for physics-based understanding, and to effect new-vehicle designs led eventually to the decision to fund and implement the proposed flight test.

It should be noted that the flight experiment received strong advocacy from the SSP Manager, the Orbiter Project Office manager, and the NASA Engineering and Safety Center. It is interesting to note that strong Program Manager advocacy was also required for the first five flights of the SSP, which served as the initial Orbiter flight tests.

Participation in the planning and execution of the flight tests included personnel from Johnson Space Center, Kennedy Space Center, Ames Research Center, Langley Research Center, the United Space Alliance, and the Boeing Company. Disciplines involved in the flight experiment included hardware, ground operations, aerothermodynamic, aerodynamic, flight control, thermal analysis, structural analysis, trajectory design, operations, loads \& dynamics, impact test \& analysis, instrumentation, avionics, software, materials \& processes, manufacturing, Safety \& Mission Assurance, and robotics experts. Representatives from the Astronaut Crew Office also participated.

Because the number of flights on the remainder of the SSP manifest was limited, implementation of the flight test required rapid planning. In order to accelerate planning, the Systems Requirements Review (SRR) and Preliminary Design Review (PDR) were held concurrently in June 2007 to formally define the flight test scope and review initial 
plans. A Critical Design Review (CDR) was held in January 2008 in Huntington Beach, CA. In order for the flight experiment protuberance and augmented instrumentation to be installed, approval was required through nearly every level of SSP management. Re-entry for the first flight of the experiment, STS-119, occurred on March 28, 2009. Re-entry for the second flight experiment, STS-128, occurred on September 11, 2009.

An activity complementary to the BLT FE led by NASA Langley was also undertaken to image the Orbiter during re-entry using infrared detectors. The Hypersonic Thermodynamic Infrared Measurements (HYTHIRM) team imaged the Orbiter on both STS-119 and STS-128 (in addition to STS-125). HYTHIRM activities are documented in References (Horvath, others).

An overview of the two flight experiments, a brief presentation of the data obtained, and a discussion about potential future flight tests on the Orbiter is presented in the sections that follow.

\section{$\underline{\text { Experiment Design Overview }}$}

\section{Protuberance Placement}

Because the Space Shuttle Orbiter is a manned vehicle, safety was the top priority in all aspects of the experiment design. For any disturbance (protuberance, cavity, etc) on the Orbiter windward surface, a wedge of turbulent flow emanates downstream of the disturbance. The flow within this turbulent wedge can increase the heating over laminar levels by several times. During each flight of the Space Shuttle, damage occurs on the Orbiter surfaces due to ascent debris. Because some areas of the Orbiter are more prone to damage due to ascent debris than others, this was taken into account in the placement of the protuberance. Damage to the protuberance due to ascent debris or more importantly damage in the turbulent wedge downstream of the protuberance could sacrifice mission success and safety. In addition to the likelihood of damage, consideration was also given to the fact that certain areas of the Orbiter have more structural capability than others. An increase in heating due to the presence of a protuberance (or other disturbance) and associated wedge could reduce structural margins due to thermal gradients. The team wanted to place the protuberance in an area that had a sufficient amount of margin to be able to withstand the increase in heating due to the turbulence.

With all these factors in mind, the protuberance location was chosen to be on the port wing, outboard and downstream of the main landing gear door (see Figure 1). Choosing to place the protuberance on the port side avoids regions of higher-risk impact sites due to the ice frost ramps and other foam sources on the starboard side of the external tank. A Systems Engineering and Integration (SE\&I) risk study was performed and found that the risk of critical damage in the turbulent wedge downstream of the protuberance was less than 1 in 10,000 . 


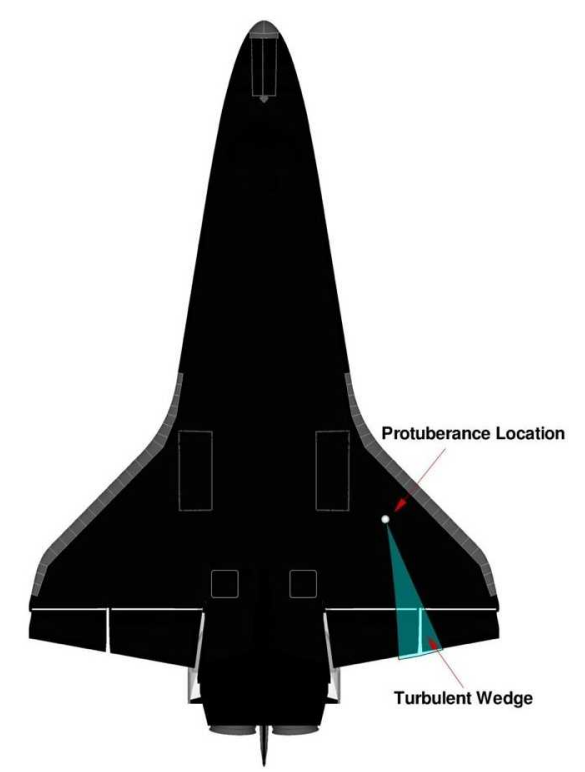

Figure 1. Selected protuberance location and predicted turbulent wedge

\section{Protuberance Design}

An incremental approach to the flight test program was decided upon early in the planning stages in order to ensure safety given the uncertainties in the prediction of BLT onset and associated effects on the TPS. As a point of reference, the earliest experienced BLT on the Orbiter occurred at approximately Mach 18 during the re-entry of STS-28. The first flight targeted a BLT onset Mach number of approximately 15 . The second flight targeted a BLT onset Mach number of approximately Mach 18. The height of the protuberance was derived using the Orbiter BLT Tool (Version 2) with the $\operatorname{Re}_{\theta} / \mathrm{M}_{\mathrm{e}}$ correlation using the best estimate correlation coefficient. See Reference (Ref. Berry 2010 paper) for more information about Version 2 of the BLT Tool. The heights for STS119 and STS- 128 were 0.25 " and $0.35 "$, respectively.

A sensitivity study was performed to understand the effect of trajectory on predicted BLT time. The range of BLT onset Mach numbers predicted for a sample of trajectories are shown in Table 1 using the STS-119 protuberance height. The Best Estimated Trajectory (BET) shown for each applicable mission was derived from Orbiter navigation data.

Table 1. Predicted BLT onset Mach numbers for a 0.25 " protuberance

\begin{tabular}{|l|c|}
\hline Trajectory & BLT Onset Mach Number \\
\hline Generic ISS Return & 16.6 \\
\hline STS-114 BET & 15.7 \\
\hline STS-115 BET & 15.2 \\
\hline STS-116 BET & 15.2 \\
\hline STS-117 BET & 14.9 \\
\hline STS-118 BET & 15.6 \\
\hline STS-120 BET & 15.3 \\
\hline STS-121 BET & 15.7 \\
\hline
\end{tabular}


Aerothermal, thermal, and stress analysis was performed for the protuberance, the tiles immediately surrounding the protuberance, and the downstream tiles within the turbulent wedge. Aerothermal analysis was performed using nominal Orbiter heating predictions from XF0002. XF0002 is briefly described in Reference (Chuck's 2006 AIAA paper) and is used to provide baseline "smooth" Orbiter heating environments. Heating augmentation factor predictions, due to the protuberance and vortex heating caused by the protuberance, were derived using computational fluid dynamics (CFD). The Data Parallel Line Relaxation (DPLR) code (Ref. DPLR reference) was used to for the augmentation factor predictions. Details on the specific analyses performed in support of the flight experiment are discussed in Reference (Tang 2010). Supporting arc jet testing and test environment calculations are detailed in Reference (Larin 2010) and (Marichalar 2010), respectively.

The baseline Orbiter heating and CFD results were combined using a heating augmentation ("bump") factor methodology. These aerothermal environments were then used as a convective boundary condition for follow-on thermal analysis. Thermal analysis to account for conduction through the TPS materials to the structure and to account for radiation between surfaces was performed using SINDA and TRASYS (references for SINDA, TRASYS, Boeing documents), respectively. Stress analysis was also performed using standard DAT tools and methods. Additional information on the analysis is presented later in this paper.

The protuberance was tile was fabricated using BRI-18 tile material. BRI-18 is an advanced, high-density tile with similar temperature capability as LI-2200, but includes more damage impact resistance capability. BRI-18 tiles are often coated with a Toughened Unipiece Fibrous Insulation (TUFI) coating that further enhances damage tolerance. For the flight experiment protuberance tile, TUFI coating was applied to all regions of the tile with the exception of the protuberance itself. The protuberance was not TUFI-coated in order to increase the likelihood that an astronaut would be able to remove the protuberance in the unlikely event of a needed repair Extra-Vehicular Activity (EVA). Repair procedures and tools developed during STS-114 were reviewed for applicability and evaluated for their ability to perform the tile protuberance removal. An illustration of this is found for a protuberance prototype in Figure 2. More information about the protuberance tile design can be found in (Ref. Spanos). 


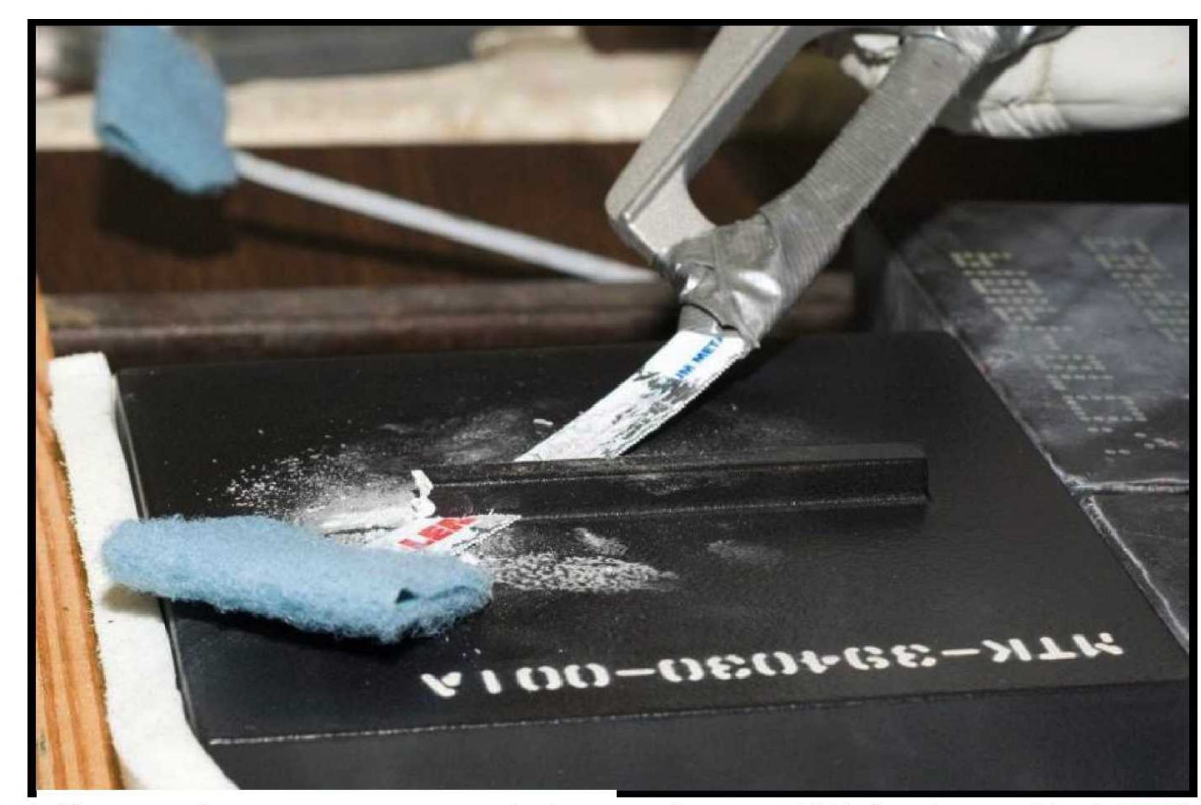

Figure 2. Tile protuberance prototype being cut by an EVA hacksaw (Ref. XX)

One of the main requirements in the design of the protuberance was that the protuberance remain shape-stable through the entry. Based on arc jet data and previous flight experience, it is expected that as a tile reaches the material capability temperature that it begins to change shape or slump. Because of this, aeroheating and thermal analysis drove the design for the protuberance shape. Nearly 10 different design iterations were assessed and two were tested in the JSC arc jet facility. The shape was designed so that heating levels would be relatively constant along the length of the protuberance. A photograph of the final design is shown in Figure 3. The length and width of the protuberance installed on the vehicle was 4.0 " and 0.376 ", respectively, for both flights. The protuberance was machined into the tile such that the leading edge was oriented at an angle of $45 \pm 3^{\circ}$ relative to the local flow streamline.

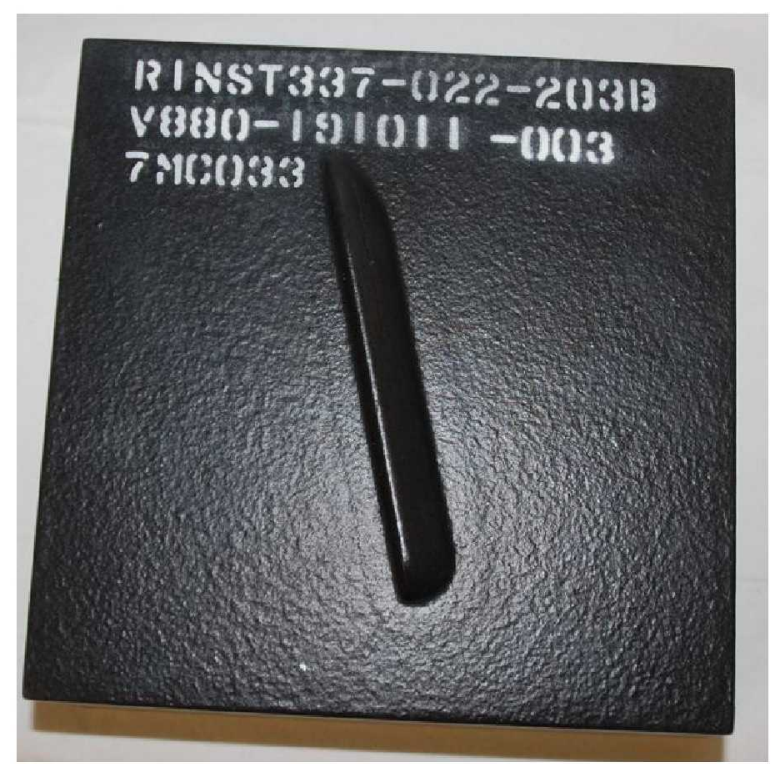

Figure 3. Final protuberance shape 
The predicted tile temperatures due to vortex heating just downstream of the protuberance drove the team to replace three of the LI-900 tiles nominally found on the Orbiter acreage with LI-2200 tile, which has a higher temperature capability. A schematic showing the different tiles in the region of the protuberance is shown in Figure 4. Additional details about the changes to the TPS can be found in Reference (Spanos paper).

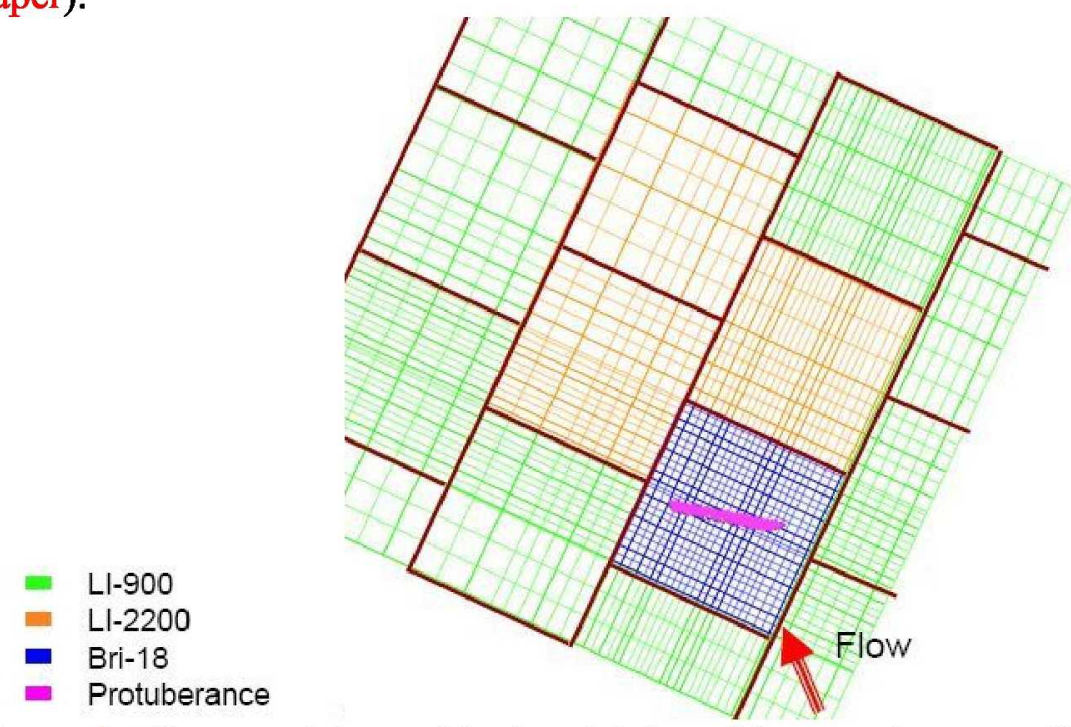

Figure 4. Tile materials used in the vicinity of the protuberance tile

\section{Catalytic Coated Tiles}

In addition to BLT and turbulent heating at high Mach number, another aerothermodynamic phenomenon that is not clearly understood is the potential coupling effect between turbulent flow and a catalytic material behavior. Current best practices in manned-spaceflight assume that turbulent and catalytic heating are additive. However, some have hypothesized that the two effects might not be additive and in fact that they would be significantly less than an additive model would predict. Because the Orion backshell TPS is being designed using fully-turbulent heating and because Orbiter tile (which is partially catalytic) has been baselined as the after-body tile material, resolution to the question about turbulent-catalytic coupling could aide in the design of Orion.

During STS-2, STS-3, and STS-5, tiles were coated with a nearly fully-catalytic material to measure the effect of catalycity on heating environments (Ref. STS-2,3,5 documents). However, these flights experienced boundary layer transition late in the trajectory where catalytic effects are not as pronounced. In order to best assess the turbulent-catalytic coupling effect, turbulence should be experienced at relatively high Mach numbers where non-equilibrium effects are more prominent. Because one of the prime objectives of BLT flight experiment was to trip the boundary layer at relatively high Mach numbers, a definite relationship to the turbulent-catalytic coupling question was apparent.

NASA JSC solicited the help of engineers at NASA-Ames who were involved in the flight experiments on STS-2, STS-3, and STS-5. A new formula for the catalytic coating was derived and tested (Ref. Spanos paper?). The new formula was developed based on 
similar material constituents as for the previous catalytic tile flight tests. The catalytic coating was then applied to two tiles placed adjacent to one another. The tiles were placed in close proximity to ensure similar environments were experienced to simplify analysis of the data following the experiment. A figure showing the coating applied to the tiles is shown in Figure 5. The apparent bumps in the figure are not as pronounced as they appear in the figure. Rather they are an artifact of the lighting in the photograph. Actual inspection of the coating showed the surface-plane deviations were minimal. Placing the coating on the tile required consideration of several constraints. These design considerations are discussed in Ref. Spanos. The catalytic coating was not applied for the first flight of the flight experiment (STS-119), but was tested on the second flight (STS128).

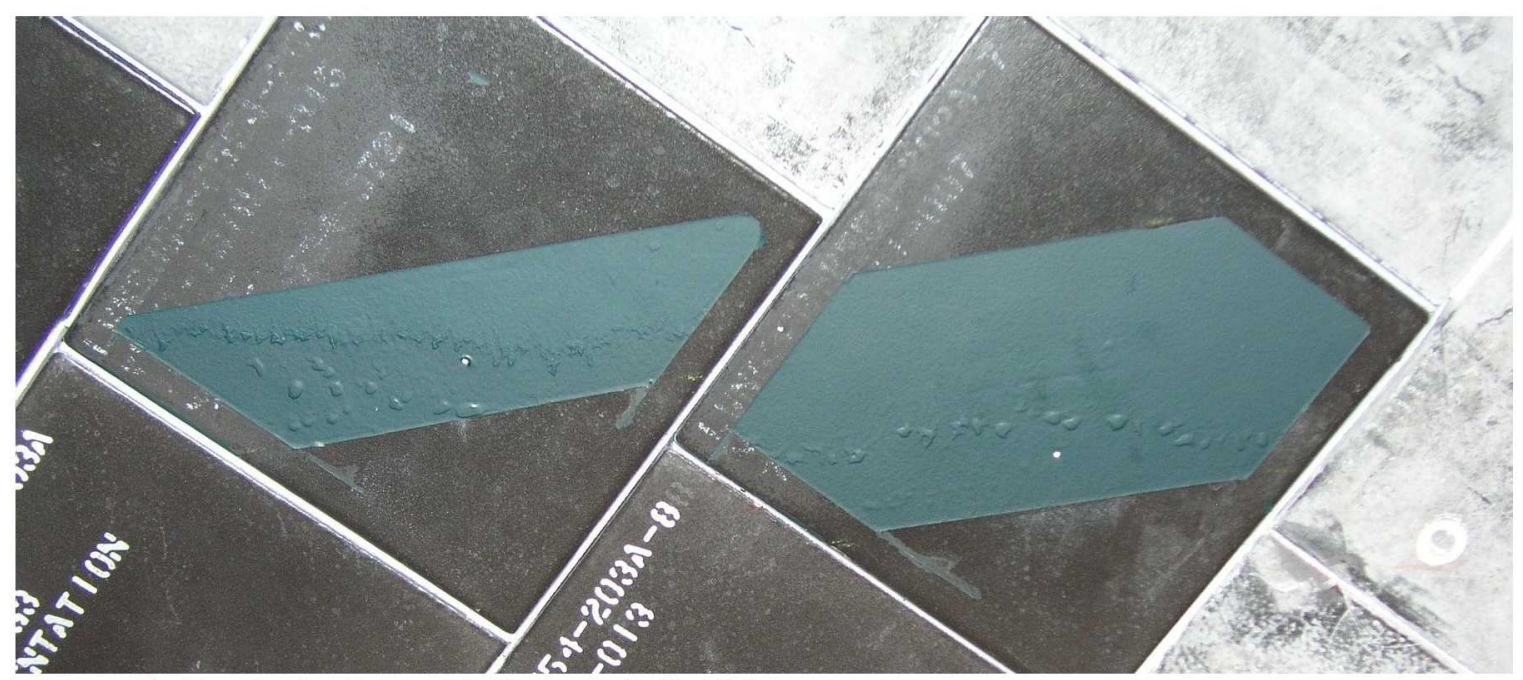

Figure 5. Catalytic Coated Tiles on STS-128.

\section{Trajectory Design}

The actual design of the Orbiter re-entry trajectory was not modified for the flight experiments. However, extensive coordination took place with flight design personnel, flight dynamics officers, and the Ascent-Entry Flight Techniques Panel to communicate potential impacts of Orbiter maneuvers on the flight experiment data. During entry the Orbiter goes through a series of roll maneuvers. These roll maneuvers have been hypothesized to effect BLT onset because of the angle of attack and sideslip modulation associated with them. The team established desired 'cut-off' intervals on either side of the predicted BLT onset Mach numbers in order to avoid roll reversals during that period of the re-entry. A flight rule related to the flight experiment was established that allows for de-orbit burn modification to occur if the cut-off intervals are violated for the primary entry opportunity on the first day option for return. In practice, these intervals did not drive mission decisions during STS-119 or STS-128.

\section{Instrumentation Overview}

Prior to installation of the flight experiment hardware, OV-103 had a total of 6 existing surface thermocouples on the windward surface of the vehicle. None of these previously available measurements were located downstream of the protuberance in the expected 
turbulent wedge. In order to adequately capture the heating levels and extent of the turbulent wedge, additional instrumentation was required. Exact placement of the thermocouples, especially for the instruments furthest downstream from the protuberance, was carried out using the wedge tool (Ref. McGinley San Francisco, Kirk Wedge) using a wedge half angle of $7.5^{\circ}$. The wedge tool is an analytical tool that uses predicted streamlines from CFD solutions to estimate the geometric extent of the turbulence downstream of a disturbance.

Figure 6 shows a sketch of the location of the instrumentation for STS-119 and STS-128. White symbols represent instrument locations active for both STS-119 and STS-128. Because some of the instruments were not used for both flights, instruments that were active for STS-119 but not active for STS-128 are colored purple. Thermocouples for STS-128 that were not active on STS-119 are colored green. The catalytic coated tiles have boxes surrounding their location. It is important to note that the flight experiment team was limited to 10 additional thermocouple locations because of data acquisition system (DAS) constraints in the wing region. Three holes were drilled into the Orbiter wing structure to run thermocouple lead wires between the DAS hardware and the outer mold line (OML) of the vehicle. Additional information about the instrumentation system can be found in Reference (Spanos paper).

The differences in instrumentation layout between STS-119 and STS-128 were implemented to (1) obtain higher resolution on the turbulent wedge and (2) to take measurements in the two catalytic coated tiles during STS-128. The catalytic coated tiles were placed as near to the center of the predicted wedge as possible and were placed adjacent to one another. The thermocouples were placed in the tiles in different locations relative to the leading edge of the coating application. On one tile the thermocouple was placed as close to the leading edge as possible while the other tile included a thermocouple in the middle of the coating application. The thermocouples were placed in this manner to measure the catalytic jump due to a mismatched material (Ref. Marichalar AIAA paper) and to measure the general catalytic behavior of the coating, respectively. 


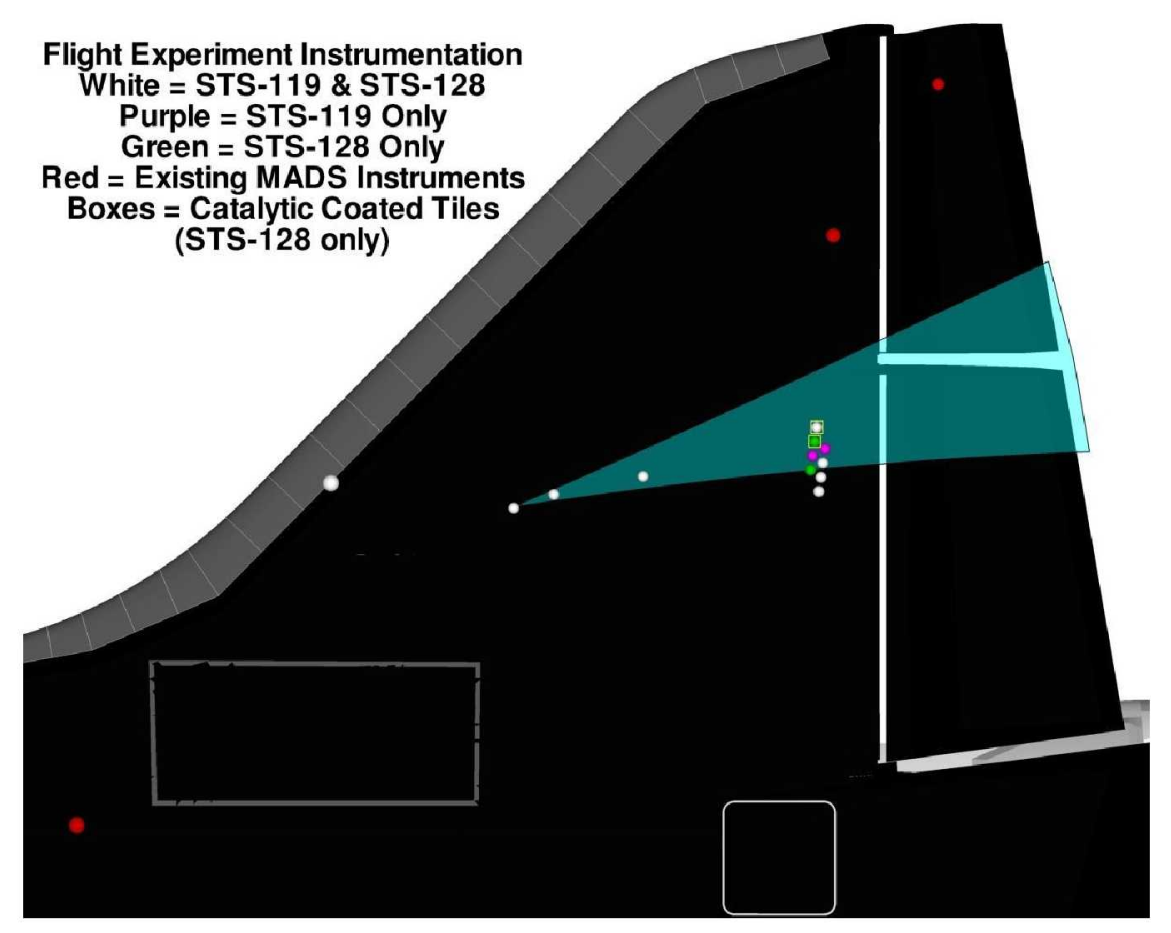

Figure 6. Instrumentation locations for STS-119 and STS-128. The turbulent wedge assumes a $7.5^{\circ}$ half-angle.

For the purposes of comparison to HYTHIRM data and computational predictions, the uncertainty levels associated with the thermocouple measurements were assessed. The components that contribute to the overall uncertainty include a precision uncertainty due to the bit resolution and recording steps in the measurement system, a MADS stability precision uncertainty, and a calibration curve precision uncertainty. A combination of these precision uncertainties is estimated at $\pm 20^{\circ} \mathrm{F}$. In addition to this precision uncertainty, there is also a known bias uncertainty due to installation effects. It is estimated that the actual tile surface temperature is approximately $20^{\circ} \mathrm{F}$ hotter than the values reported by the thermocouple measurement due to the thermocouple placement slightly below the tile outer mold line. In actuality, this bias effect is measurement location dependent as each thermocouple is installed at a slightly different depth. Team members plan to perform additional analysis in the future to more precisely characterize this bias effect.

\section{STS-119}

\section{Pre-flight STS-119 Analytical Predictions}

To ensure safety of the crew and vehicle, pre-flight predictions for the area downstream of the protuberance were performed for two general regions. The first region considered the area from the protuberance and aft to the end of the vehicle. A graphical representation of the area is illustrated in Figure 1, where the areas within the turbulent 
wedge and surrounding the turbulent wedge were analyzed. This analysis considered the global effects of the turbulent heating within the wedge on thermal and structural margins. The results of this more global analysis were performed using standard Orbiter analysis tools. These analyses for early BLT have shown that an undamaged Orbiter maintains acceptable thermal and structural margins for BLT up to Mach 21 on the wing, even when considering the thermal gradients that exist across the turbulent wedge.

The second analysis region was more limited in geometrical scope and only considered the area immediately surrounding the protuberance. This area is shown pictorially in Figure 4 . The intent of this analysis domain was to capture any adverse local effects due to the protuberance and associated vortex heating on the thermal and structural margins. As discussed previously, the local aeroheating analysis was carried out using a combination of XF0002 and CFD. The CFD solutions were provided using the DPLR code for select trajectory points and are discussed in Ref (Tang). Thermal and structural analyses were also performed, as discussed previously.

A summary of the predicted material temperatures is presented in Table 2 for the preflight (predicted) STS-119 trajectory and a generic (conservative) ISS return trajectory, assuming BLT takes place upstream of the protuberance at approximately Mach 8 . Other trajectories were also analyzed but are not shown. In the case of the generic ISS return trajectory (which was ultimately used for certification of the experiment), a material temperature waiver was required for the protuberance as the predicted temperature of $3028^{\circ} \mathrm{F}$ exceeded the single-use limit for BRI-18 of $2900^{\circ} \mathrm{F}$ by $128^{\circ} \mathrm{F}$. Acceptable tile factors of safety and structural margins were predicted.

Table 2. Pre-flight STS-119 analysis predictions for pre-flight predicted STS-119 and generic ISS return trajectories. *Room Temperature Vulcanizing (RTV) material is used to bond TPS tiles to the strain isolator pad (SIP) and to bond the SIP to the aluminum structure.

\begin{tabular}{lcc}
\multicolumn{1}{c}{ Material } & \multicolumn{2}{c}{ Peak Temperature $\left({ }^{\circ} \mathbf{F}\right)$} \\
\cline { 2 - 3 } Protuberance & STS-1 19 Pre-Flight & Generic ISS Return \\
Protuberance tile acreage & 2892 & 3028 \\
LI-2200 tile & 2720 & 2720 \\
LI-900 tile & 2535 & 2724 \\
RTV* & 2312 & 2529 \\
Aluminum structure & 419 & 533 \\
& 152 & 187
\end{tabular}

\section{Mission Overview}

Space Shuttle Discovery launched from Kennedy Space Center (KSC) on March 14, 2009. The mission consisted of 4 spacewalks to continue construction of the ISS. The Orbiter was docked to the ISS for 10 days. During the mission, the crew delivered and installed the final ISS truss segment, known as S6. The final pair of power generating solar array wings were also delivered and successfully installed. All prime objectives of the mission were achieved and following undocking, the ISS was ready to house a sixmember crew. Discovery landed safely at KSC on March 28, 2009. 
During the mission, the Damage Assessment Team (DAT) assessed 9 items. These items included one lower surface elevon cavity damage, two tile surface discoloration sites, two minor windward surface thermal barrier frays/protrusions on the nose and main landing gear doors, three gap filler protrusions on the upper surface Orbiter Maneuvering System (OMS) pod, and one gap filler protruding on the lower left inboard elevon. With the exception of the protruding gap filler and the elevon cavity damage, relatively little effort was required to clear the sites. Aeroheating, thermal, and stress analysis was required for both the elevon sites which were eventually cleared.

A post-flight review of the thermocouple data showed a relatively early ( $\sim$ Mach 11.5 ) asymmetric BLT event on the starboard side of the Orbiter during the STS-119 re-entry. This asymmetric event was also observed in HYTHIRM imagery (Ref Horvath) as shown in Figure 7. The HYTHIRM imagery proved to be very useful and was used in conjunction with the wedge tool and the effected thermocouple locations to help isolate regions that might have caused a flow disturbance. No out-of-tolerance steps or protruding gap fillers were identified that could be attributed to the asymmetric BLT event during post-flight inspection.

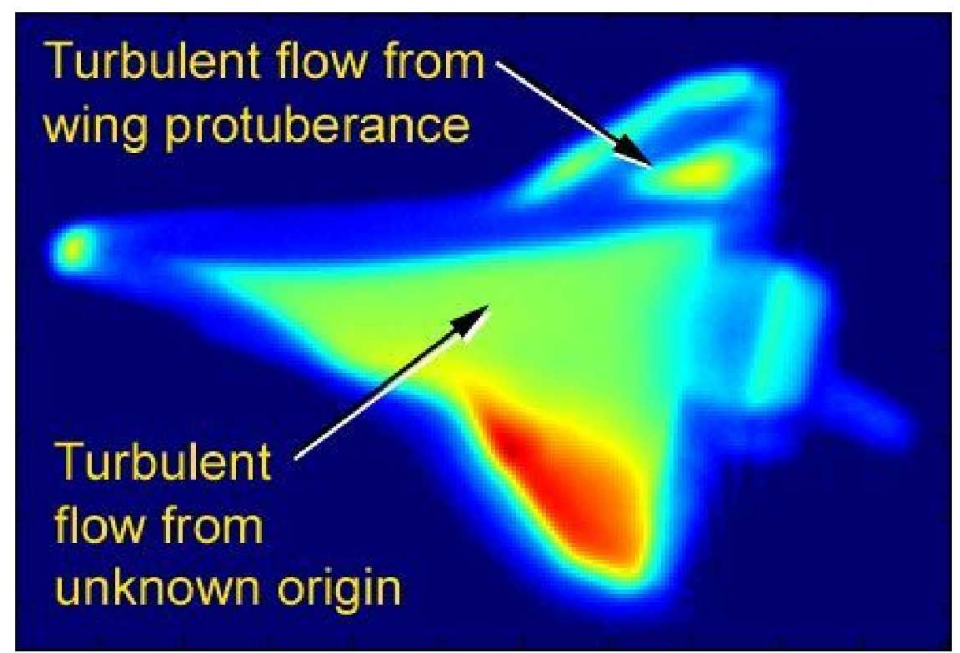

Figure 7. HYTHIRM imagery from STS-119. See Reference Horvath.

\section{Flight Data Overview}

For convenience during the discussion of the flight data, the labeled flight experiment thermocouples are shown in Figure 8. STS-119 flight data in the form of thermocouple time traces are shown in Figures 9 and 10. The thermocouple data is plotted in two different plots for the sake of clarity. Figure 9 shows the thermocouple data associated with the flight experiment thermocouples that are furthest aft, while Figure 10 shows the data for thermocouples furthest forward and on the protuberance. The data is plotted using the post-flight BET. The temperatures are plotted verses time from Entry Interface (EI). EI is defined to the time at which the Orbiter altitude is 400,000 feet. Also shown in the figures are the angle of attack and roll angle profiles for the re-entry. 


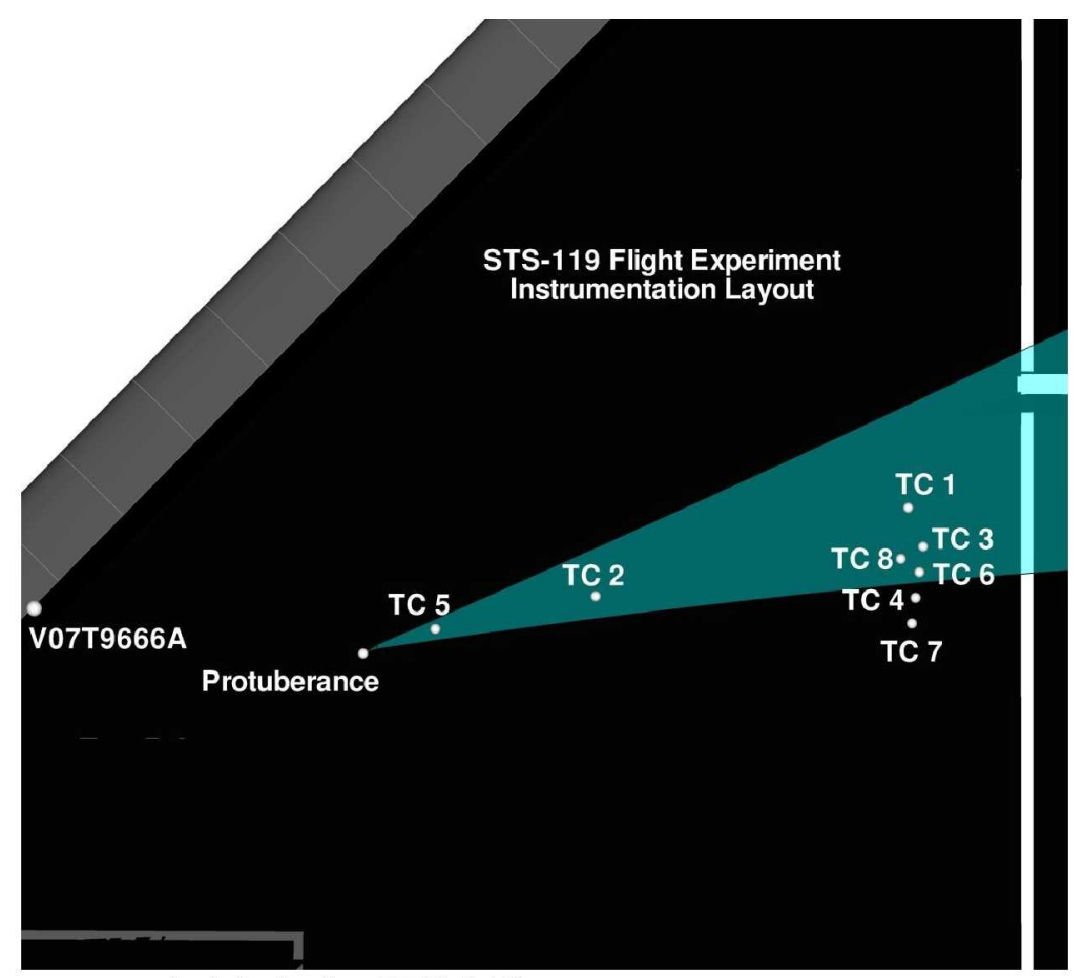

Figure 8. Thermocouple labels for STS-119.

A cursory glance at the aft thermocouple traces in Figure 9 show that temperatures did not exceed $2000^{\circ} \mathrm{F}$. Peak temperatures were experienced during the turbulent portion of the entry. Temperatures during the laminar portion of the trajectory were all similar for the thermocouples shown with peak laminar temperatures between approximately 1500 and $1600^{\circ} \mathrm{F}$. Thermocouple 1 (hereafter referred to as TC 1 ) is located at the center of the predicted wedge and shows the earliest sign of BLT between 950 and 1000 seconds. This corresponds to a Mach number of 15.6. BLT time was selected based on the earliest time that temperatures departed from laminar levels. It should be noted that the process of selecting BLT time can be somewhat subjective, depending on the data under consideration.

The thermocouple data then indicates that the turbulent wedge progresses inboard (and presumably outboard on the opposite side of the wedge), as expected, with TCs 3,8 , and 6 showing BLT very quickly following TC 1 . TCs 4 and 7 show transition times much later than the other aft thermocouples. These two thermocouples appear to have been correctly placed outside the turbulent wedge. Based on these observations, the turbulent wedge half angle is $7.0^{\circ} \pm 0.5^{\circ}$. This value was derived by drawing a straight line between TC5 back to TCs 6 and 4 and measuring the angle between that line and TC1. 


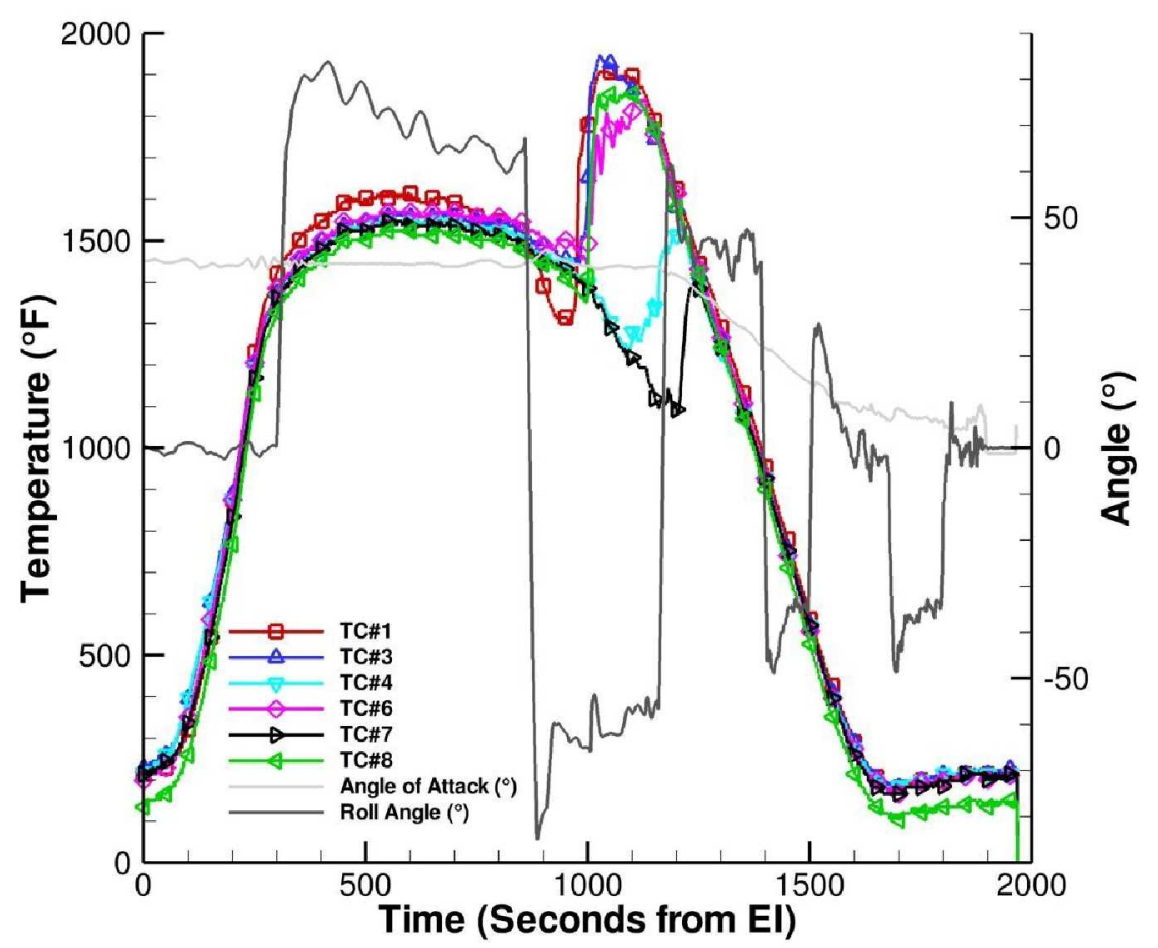

Figure 9. STS-119 data for aft thermocouples

Figure 10 shows the thermocouple traces for the forward and protuberance thermocouples. As can be seen, the maximum temperature was approximately $2000^{\circ} \mathrm{F}$ and was experienced by the protuberance. As expected, the protuberance temperature was higher than the downstream temperatures during all portions of the trajectory. In this plot, V07T9666A is located upstream of the protuberance and just downstream of the Orbiter wing leading edge. This instrument was used as a reference for interpreting the heating behavior upstream of the protuberance. Due to the location of this instrument just downstream of the wing leading edge, it is not surprising that this thermocouple registered temperatures higher than TCs 2 and 5 . TCs 2 and 5 have very similar temperatures during the laminar portion of the trajectory. The time of BLT for each of the thermocouple locations is also worth noting. As expected, TC 2 experiences BLT first, followed by TC 5 and finally the protuberance. This is consistent with the turbulent front traveling upstream with time. TC 2 is the furthest aft of the measurements shown in this plot. Since TC 5 represents the measurement closest to the protuberance, it is considered to be a measure of the time for fully effective BLT due to the protuberance. Based on the BET, TC 5 experienced BLT at Mach 13.1. The protuberance itself experienced BLT at Mach 7.7. The reference thermocouple (V07T9666A) experienced BLT at approximately Mach 8.5.

An interesting signature is observed in some of the thermocouple traces shown in Figure 10. As can be seen, at approximately 850 seconds there is a sharp drop in temperature in the protuberance and reference measurements. By examining Figure 10 the angle of attack and roll data in the same figure, one can see that this time corresponds to a vehicle maneuver in the form of a roll-reversal. Close examination of the plot also reveals a 
slight change in angle of attack associated with the roll-reversal. This thermocouple behavior associated with vehicle maneuvers has been observed on many previous Orbiter flights for the V07T9666A measurement. At approximately 900 seconds, TC 2 has an abrupt rise in temperature and then quickly drops to levels consistent with previous trends. The cause of this behavior is currently not understood.

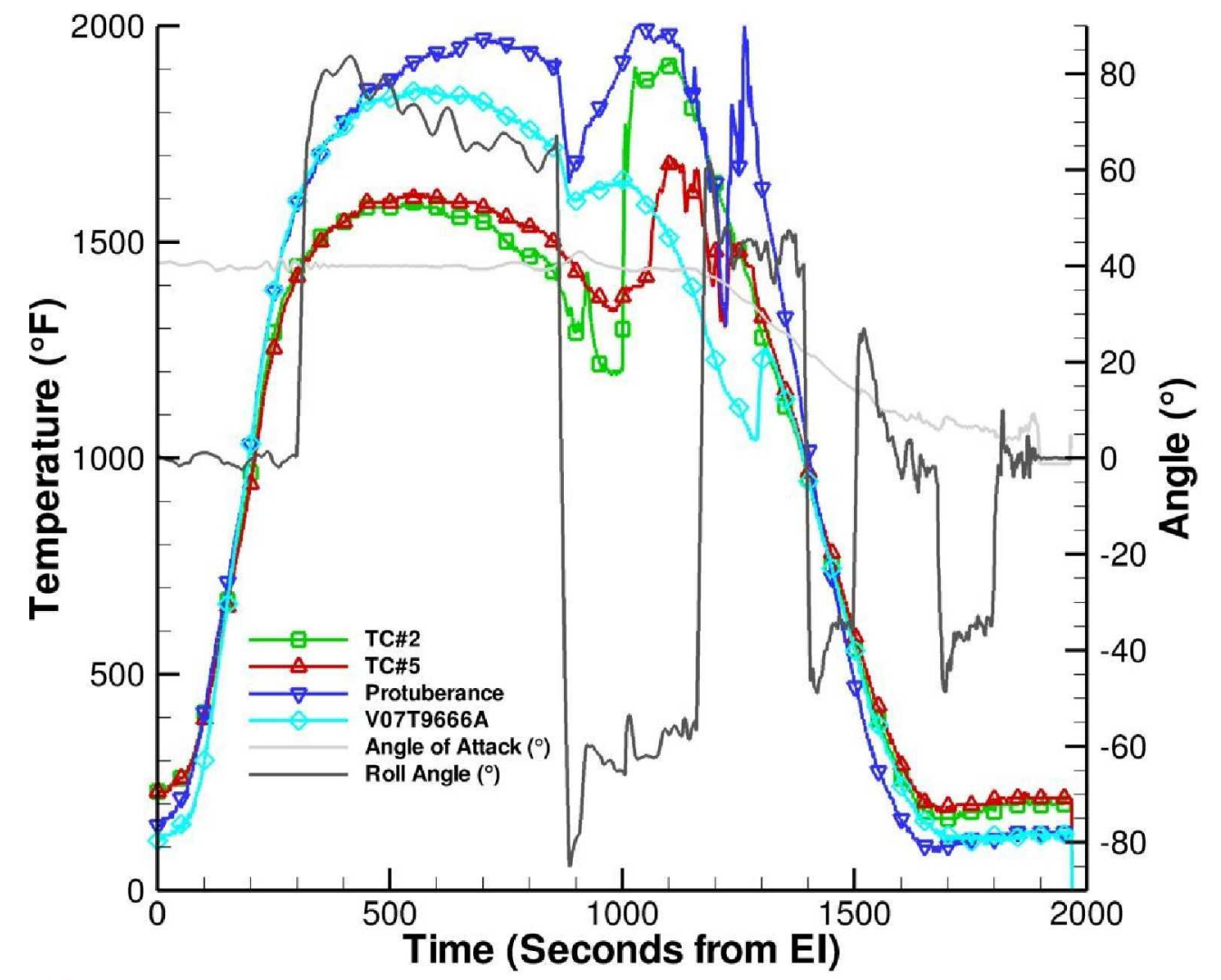

Figure 10. STS-119 data from "forward" thermocouples

Following the flight of STS-119, the tiles downstream of the protuberance were examined by TPS and material experts. Based on expert opinion and experience with the behavior of tile coating in arc jet facilities, it was determined none of the tiles effected by the flight experiment exceeded temperatures of $2500^{\circ} \mathrm{F}$. However, some of the tiles did exhibit deposits that provided clues as to the vortex heating structure downstream of the protuberance. See Figure 11. Because of these observations, it was concluded that TC 5 was not actually located in the vortex-heating region as originally desired. Using these post-flight observations and predicted heating distributions provided by CFD, the thermocouple was relocated for STS-128. 


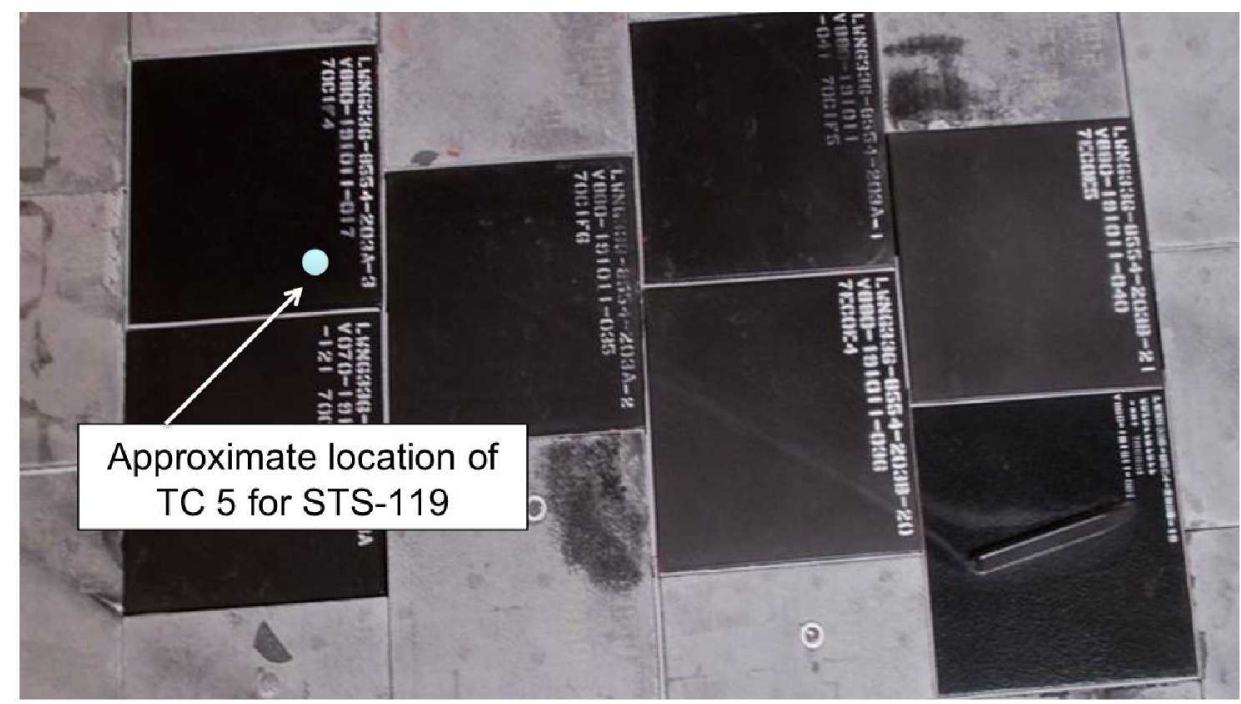

Figure 11. STS-119 post-flight photo showing streamline structure

The protuberance tile was removed from the vehicle in a non-destructive manner for further evaluation and sent to material experts for additional study. The tile was scanned pre- and post-flight using an Optigo measuring device to assess the protuberance shape change. Comparing the pre- and post-test scan results shows that the protuberance did not change shape appreciably. The observed geometry changes were between 0.0074 and 0.0212 inches, depending on location. The uncertainty in this measurement is not currently characterized because of difficulties in alignment when the measurements were taken.

\section{Flight Data and Analysis Prediction Comparisons}

Because analysis would play such a vital role in providing rationale for the safety of any follow-on flight experiment, extensive comparisons between the STS-119 flight data and analysis predictions were performed. These comparisons included the predicted BLT onset time versus observed onset time and predicted material temperatures versus observed temperatures.

Comparisons between predicted and earliest observed BLT onset times were very favorable. As discussed in Ref (Berry), the predicted onset time for a 0.25 -inch height using the BET was Mach 15.4. The observed BLT onset time at TC 1 was Mach 15.6. This is within the $\pm 1-\sigma$ prediction uncertainty of the BLT Tool.

Comparisons between predicted and observed temperatures were not as favorable as the BLT onset time comparisons. Of particular note was the predicted temperature on the protuberance $\left(2892^{\circ} \mathrm{F}\right.$ based on the pre-flight STS-119 trajectory) versus the maximum reported temperature on the protuberance $\left(2011^{\circ} \mathrm{F}\right)$. This nearly $900^{\circ} \mathrm{F}$ difference equates to a difference in heat flux of over a factor of four. The ability to compare predictions to actual flight data was less precise for the tiles surrounding the protuberance because of the sparseness of the instrumentation. However, material experts stated that the tile coating temperature did not exceed $2500^{\circ} \mathrm{F}$. This is in contrast to the maximum pre-flight prediction of $2720^{\circ} \mathrm{F}$ for the protuberance tile acreage. This represents a difference in 
heat flux of at least 1.4 times. It is, however, believed that the temperatures were actually much lower than $2500^{\circ} \mathrm{F}$. The cause of the large discrepancy between predicted and observed temperatures is currently unknown.

\section{Analysis in Support of Flight Experiment \#2}

In order to ensure that the second flight of the flight experiment (using a protuberance height of 0.35 -inches) would not impact safety or vehicle integrity, additional analysis was required. In considering the analysis performed, it is important to point out two important facts: (1) no readily-available explanation for the large discrepancy between prediction and observation on STS-119 was determined and (2) due to the flight manifest, a very quick decision was needed as to whether or not a protuberance tile should be installed and if so, what height should be installed. In actuality, the team had on the order of 1 month to review the STS-119 data, revise models, and perform an adequately rigorous analysis to certify the safety of the crew and vehicle.

Because no readily available explanation had been determined to explain the analysis discrepancy, an ad-hoc approach was selected to create "knockdown" factors in an attempt to match the STS-119 data. The team felt relatively confident in the ability of the CFD predictions to accurately describe the distribution of heating. However, the magnitude of the combination of the undisturbed and heating augmentation factors was in question. Following their derivation, the knockdown factors were then applied to the STS-128 pre-flight certification analysis with the assumption that the knockdown factors were appropriate for use with the 0.35 -inch protuberance. While 0.25 -inch protuberance CFD solutions were used for the STS-119 reconstruction and knockdown factor derivation, 0.35-inch CFD solution heating distributions were used for the STS-128 certification analysis (see Ref. Tang). These distributions were used in combination with the undisturbed heating, CFD heating augmentation factor magnitudes, and derived knock-down factors. The relationship between the different parameters was described by Equation 1 and Equation 2.

$$
\begin{array}{cr}
\dot{q}=\dot{q}_{X F 0002}\left[\left(B F_{C F D}-1\right) k+1\right] & \text { Equation 1 } \\
\dot{q}=\dot{q}_{X F 0002}\left[B F_{C F D} \cdot k\right] & \text { Equation 2 }
\end{array}
$$

In these equations, $\dot{q}$ represents the heat flux applied, $\dot{q}_{X F 0002}$ represents the undisturbed smooth-body Orbiter heating predicted by XF0002, $B F_{C F D}$ is the unadjusted heating augmentation ("bump") factor predicted by CFD, and $k$ is the derived knock-down factor.

For the STS-128 analysis, the BLT time needed for the prediction was derived using a generic ISS return trajectory, the protuberance height of 0.35 -inches, and the $\operatorname{Re}_{\theta} / \mathrm{M}_{e}$ correlation in version 2 of the BLT tool (Ref. Berry 2010). In order to accurately describe the heating immediately downstream of the protuberance, a fully effective BLT time was required. This time was derived using available fully effective wind tunnel data correlations that were calibrated to the STS-119 fully effective BLT time. The formulation of the correlation and the calibration to the flight data were very similar to that performed for the BLT tool (Ref. Berry 2010). CFD solutions were available at 
Mach numbers of 20,18, 15, and 12. Linear interpolation was carried out on the values for $k$ and $B F_{C F D}$ for points in the trajectory between the available CFD solutions.

Results of the analysis are shown in Table 3. It should be noted that the analysis associated with the STS-128 pre-flight trajectory assumed that a disturbance upstream of the BLT FE protuberance caused BLT on the BLT FE protuberance to occur at Mach 16.2. The analysis also limited the baseline Orbiter turbulent heating based on observations from STS-119, STS-121, and STS-28. The results for the Generic ISS Return trajectory assume a nominal ( Mach 8 ) upstream transition.

In the case of the generic ISS return trajectory (which was used for certification of STS128), a material temperature waiver was required for the protuberance as the predicted temperature of $2925^{\circ} \mathrm{F}$ exceeded the single-use limit for BRI-18 of $2900^{\circ} \mathrm{F}$ by $25^{\circ} \mathrm{F}$ for approximately 25 seconds. Rationale for the appropriateness of the waiver was based on the fact that (1) the predicted time of the over-temperature condition was relatively short (2) a certification-level (conservative) trajectory was being used for the assessment and (3) despite the surface over-temperature condition, acceptable tile factors of safety and structural margins were predicted. It was also believed that the analysis was still conservative, even after the adjustments made following STS-119.

Table 3. Pre-flight STS-128 analysis predictions for pre-flight predicted STS-128 and generic ISS return trajectories.

\begin{tabular}{lcc}
\multicolumn{1}{c}{ Material } & \multicolumn{2}{c}{ Peak Temperature $\left(^{\circ} \mathbf{F}\right)$} \\
\cline { 2 - 2 } Protuberance & STS-128 Pre-Flight & Generic ISS Return \\
Protuberance tile acreage & 2799 & 2654 \\
LI-2200 tile & 2995 & 2925 \\
LI-900 tile & 2887 & 2890 \\
RTV* & 2450 & 2475 \\
Aluminum structure & 444 & 489 \\
& 161 & 186
\end{tabular}

\section{STS-128}

\section{Mission Overview}

For STS-128, Space Shuttle Discovery launched from Kennedy Space Center on August 29,2009 . The mission consisted of 3 spacewalks and the Orbiter was docked to the ISS for 9 days. The three spacewalks consisted of, among many other things, replacing experiments outside the European Space Agency's Columbus laboratory and installation of a new ammonia storage tank for ISS cooling. The Orbiter also carried with it the 4.5ton Multi-Purpose Logistics Module (MPLM), which was filled with supplies for the ISS. All prime objectives of the mission were achieved. Discovery landed safely at Edwards Air Force base in California on September 11, 2009.

During the mission, the Damage Assessment Team (DAT) assessed 13 TPS discrepancies. Nearly half $(6)$ of these were cleared using previously performed 
bounding analyses. Of the 13 , three were tile damage cavities assessed with depths provided by photogrammetry. Incidentally, two damages were found to have occurred on either side of the flight experiment protrusion (see Figure 12). A frayed blanket at the forward Orbiter-external tank attach point was determined to have no significant effect on BLT because the fray was expected to bend during entry. Two OMS pod gap fillers and one Felt Reusable Surface Insulation (FRSI) plug were also examined and cleared by previous analysis and experience.

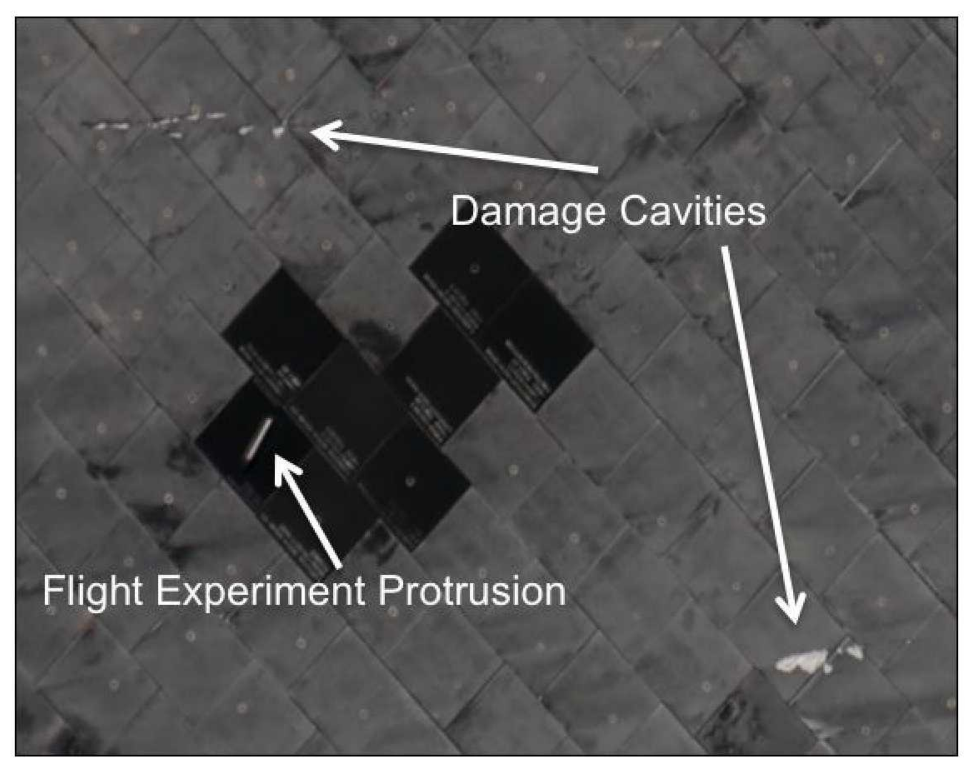

Figure 12. Damage cavities near flight experiment protrusion on STS-128.

Similar to STS-119, following Discovery's landing and upon examination of the thermocouple data, it was clear that Discovery had again experienced an early asymmetric BLT event. Unfortunately for the flight experiment, this asymmetric event took place on the port wing upstream of the flight experiment protuberance and associated thermocouples. Figure 13 is a graphic showing the turbulent wedge for an assumed disturbance location in addition to the thermocouples and associated BLT Mach numbers. The asymmetric event initiated at approximately Mach 13.6. The effects of this upstream event unfortunately make it challenging to interpret the flight experiment data in some instances. HYTHIRM observations at closest approach were earlier than the measured BLT times and so observations of the asymmetric wedge similar to STS-119 are not available as shown in Figure 14. Also similar to STS-119, the exact cause of the early asymmetric BLT event is unknown notwithstanding a focused effort to inspect the vehicle for out of specification steps, gaps, and for missing gap fillers. 


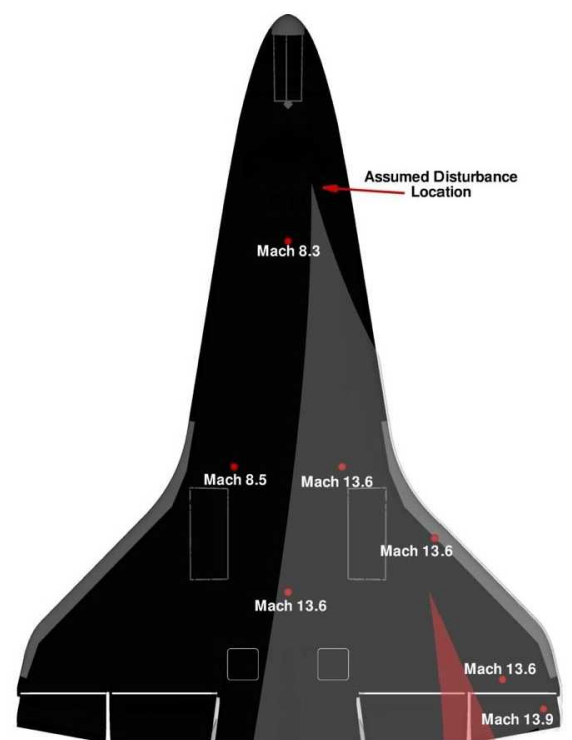

Figure 13. Post-flight reconstruction of asymmetric BLT event.

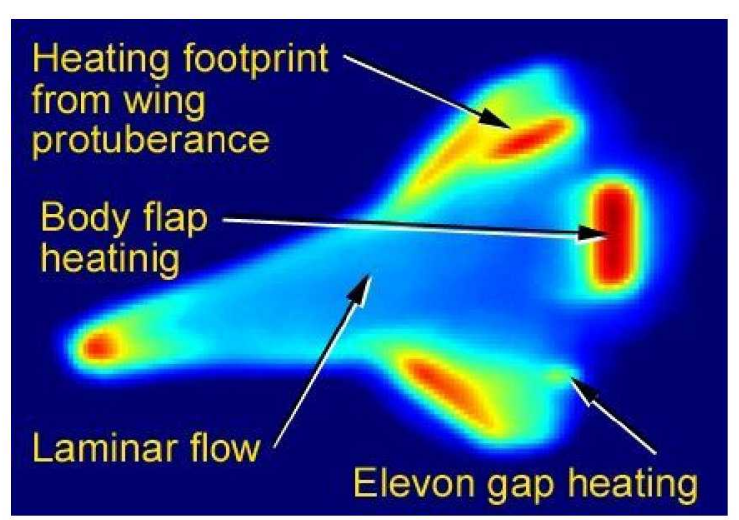

Figure 14. HYTHIRM imagery from STS-128 (Ref. Horvath)

\section{Flight Data Overview}

For convenience during the discussion of the flight data, the labeled flight experiment thermocouples are shown in Figure 15. STS-128 flight data in the form of thermocouple time traces are shown in Figures 16 and 17. The thermocouple data is plotted in two different plots for the sake of clarity. Figure 16 shows the thermocouple data associated with the flight experiment thermocouples that are furthest aft, while Figure 17 shows the data for thermocouples further forward and on the protuberance. The data is plotted using the post-flight BET. The temperatures are plotted verses time from Entry Interface (EI). 


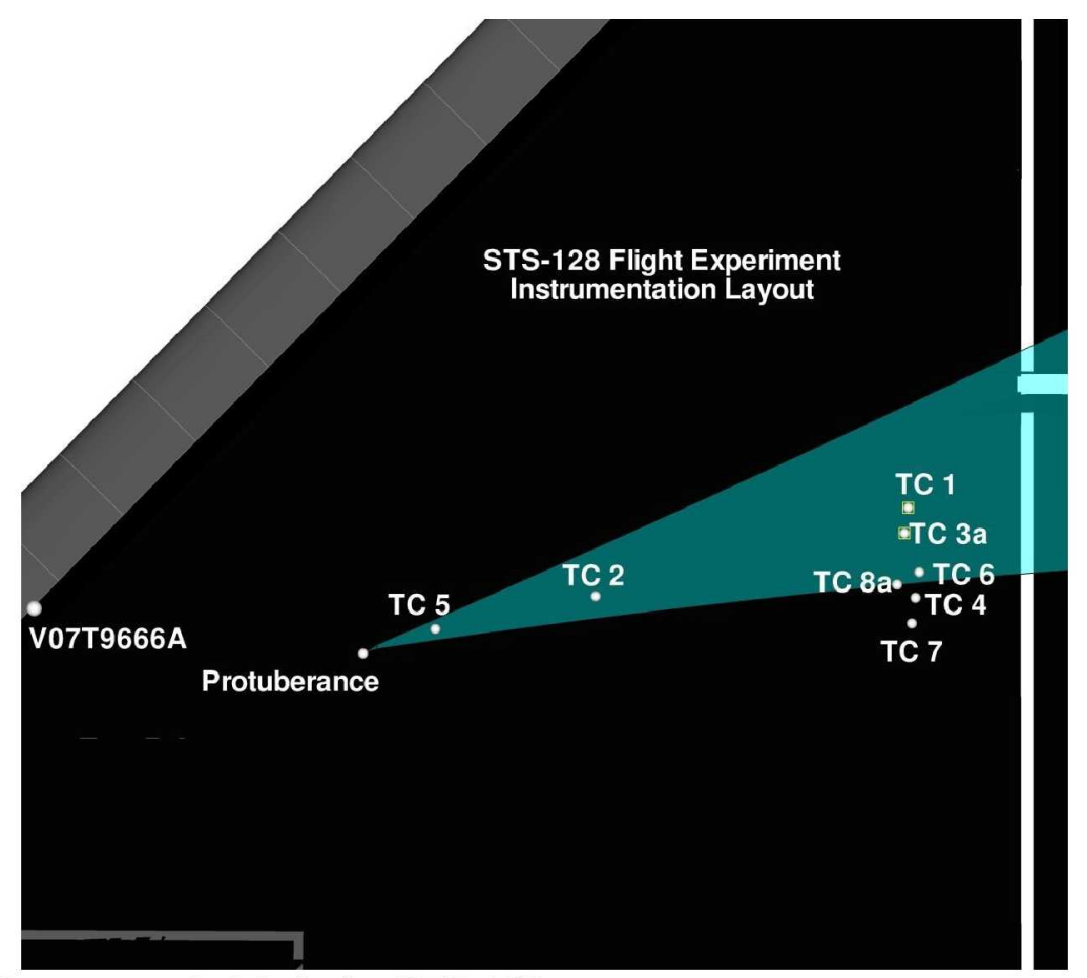

Figure 15. Thermocouple labels for STS-128.

A cursory glance at the aft thermocouple traces in Figure 16 show that temperatures did not exceed $2000^{\circ} \mathrm{F}$. Peak temperatures were experienced during the turbulent portion of the entry. With the exception of TC 1 and TC 3a, temperatures during the laminar portion of the trajectory were all similar with peak laminar temperatures between approximately 1500 and $1600^{\circ} \mathrm{F}$. In fact, the traces for TC 4, TC 6, TC 7, and TC 8a lie nearly on top of each other through the entire re-entry. It should be noted again that both TC 1 and TC 3a had catalytic coating applied to the surface. As such, the elevated temperatures in relation to the other aft thermocouples is not unexpected. It is also worthwhile to note that because of the placement of the thermocouple at the leading edge of the catalytic coating, it was also expected that TC 3 a would exhibit a higher temperature than TC 1 . The magnitude of the temperature levels and the trends with time for TC 1 and TC 3a, however, are somewhat troubling. While a physics-based explanation for the drop in temperature during the laminar portion of the trajectory is possible due to the reduction in non-equilibrium heating potential as the altitude and Mach number are reduced, the behavior of TC 1 and TC $3 a$ during the turbulent portion of the re-entry is not understood. As can be seen, the traces appear to be very noisy. Additional, more detailed assessments of the heating in Stanton number space (not shown) also cast some doubt on the measurement validity, especially in the turbulent regime. This was a disappointment to the flight test team since obtaining coupled turbulent-catalytic heating behavior at high Mach numbers was one of the prime objectives of the STS-128 BLT FE. 


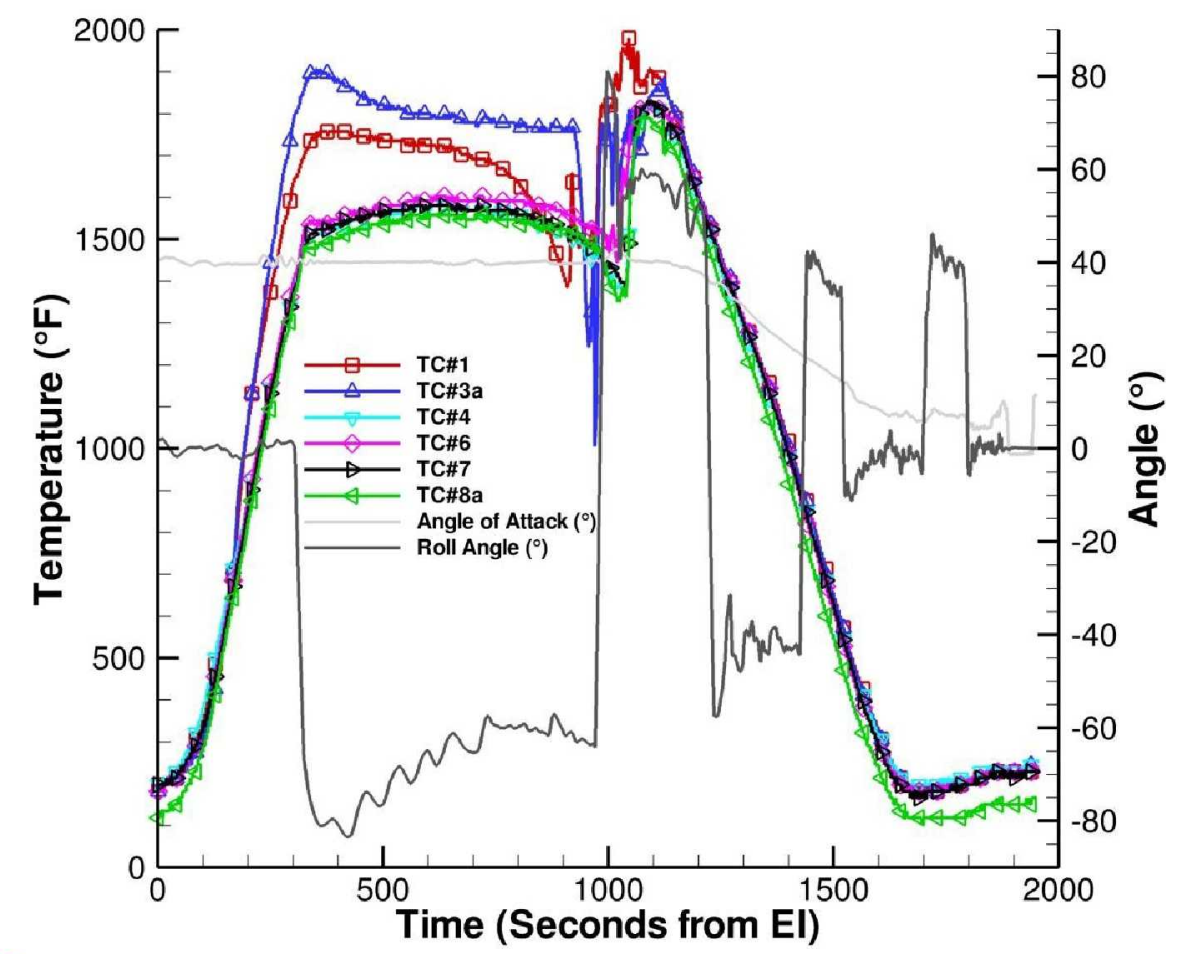

Figure 16. STS-128 data for aft thermocouples

Consistent with STS-119, TC 1 is located at the center of the predicted wedge and shows the earliest sign of non-laminar behavior at 913 seconds. This corresponds to a Mach number of 17.5 based on the BET. The thermocouple data then indicates that the turbulent wedge progresses inboard, as expected, with TC 3 a showing the next sign of non-laminar behavior. However, there appears to be a delay in BLT of approximately 60 seconds between TC $3 \mathrm{a}$ and TCs $6,8 \mathrm{a}, 4$, and 7. Based on this information, it appears that the turbulent wedge was initially very narrow before widening later in the trajectory. It is important to note, however, that the upstream asymmetric BLT event most certainly effected wedge observations. This is evidenced by the fact that during STS-119, TCs 4 and 7 showed transition times much later than the other aft thermocouples, indicating that they were outside the turbulent wedge. In this case, TC 4 and TC 7 appear to have experienced transition due to the asymmetric event although it is hard to draw conclusions as to whether or not BLT at these locations was first caused by the BLT FE protuberance or by the unknown upstream disturbance. Based on these observations, the turbulent wedge half angle was not formally assessed.

Figure 17 shows the thermocouple traces for the forward and protuberance thermocouples. As can be seen, the maximum temperature from the measurements was just below $2600^{\circ} \mathrm{F}$ and was experienced by the protuberance. Examination of the thermocouple traces for these forward thermocouples shows unexpected behavior for several of the thermocouples.

During the early portion of the trajectory it can be seen that the protuberance thermocouple exhibits the highest temperature and has a laminar peak relatively early in the re-entry at approximately 350 seconds. Unexpectedly, the temperature then steadily 
decreases until rising again after reaching a temperature of about $1300^{\circ} \mathrm{F}$ at approximately 850 seconds. Just before 1100 seconds, the thermocouple signal drops-out completely. It then rises to the maximum temperature (presumably a turbulent value) at around 1150 seconds. Protuberance BLT was assessed to have taken place at 1041 seconds, or Mach 13.6 due to the upstream disturbance. One observation that leads to the conclusion that the protuberance thermocouple is suspect during the laminar portion is that the temperature during the laminar portion for STS-128 is lower (for a 0.35 " protuberance) than the laminar temperature for STS-119 (for a 0.25 " protuberance). This observation cannot be explained from a fluid dynamics perspective. However, post-flight examination of the protuberance surface by material experts seems to corroborate the maximum $\left(\sim 2600^{\circ} \mathrm{F}\right)$ temperature measured by the thermocouple due to changes in the tile surface.

TC 5, located just aft of the protuberance, experiences a continual rise in temperature over the course of the early portion of the trajectory. This behavior is attributed to the relocation of the measurement into the vortex-scrubbing region. Presumably, this vortex heating interaction to the surface masks any clear interpretation of the BLT time, although it was estimated at 1021 seconds, or Mach 14.2. The small spike immediately prior to 1021 seconds is attributed to a vehicle maneuver.

TC 2 exhibits similar behavior to the protuberance thermocouple. It reaches a laminar peak relatively early in the trajectory and then steadily decreases before a rapid rise in temperature. The rapid rise in temperature is assumed to have been caused by transition as a result of the BLT FE protuberance. The reference thermocouple, V07T9666A, seems to exhibit relatively nominal behavior in contrast to the discontinuous behavior exhibited on STS-119.

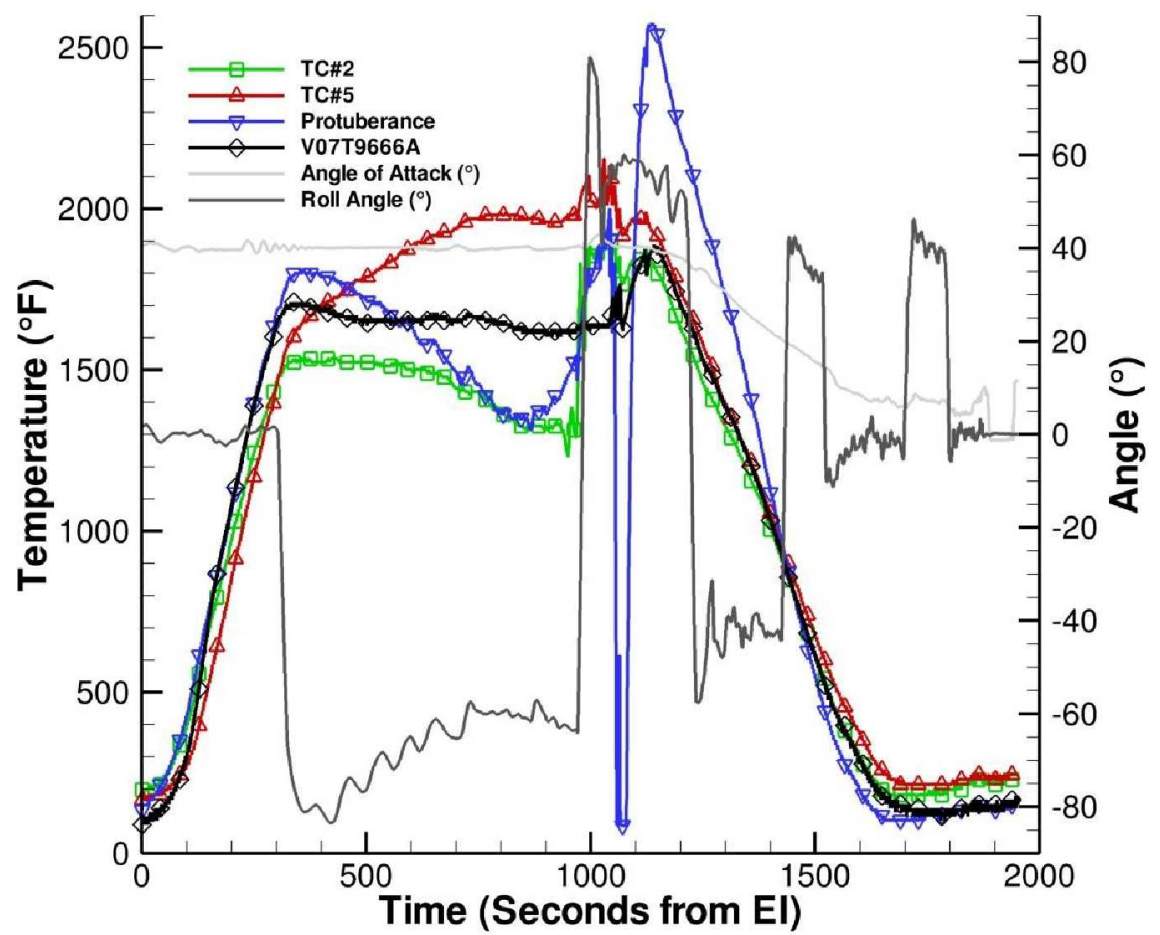

Figure 17. STS-128 data for forward thermocouples 
The cause for the measurement anomalies observed on STS-128 is currently unknown, although similar behavior has been seen on previous Orbiter re-entries. The V07T966A measurement seems to exhibit the unexpected behavior most often of any of the fleet thermocouples, although it has been observed on nearly all tile surface temperature measurements (wing, fuselage, OMS pod). Preliminary studies have shown a correlation with roll angle. Ground operations and instrumentation personnel have assessed the system but have found nothing out of the ordinary. It is most often assumed that the thermocouple circuit has intermittent connectivity or that the measurement is real and there is an unexplained aerodynamic or fluid dynamic effect causing the observed behavior in the data. Additional hypotheses are also being considered. These include Radio Frequency (RF) coupling due to the 'antenna' arrangement of the tile thermocouples, a direct voltage or current coupling flow to the thermocouple circuit, and a semi-conductor based Schottky diode effect. The flight experiment team has been coordinating with the Orion technical community in an attempt to prevent anomalous, non-real measurements for Orion re-entry.

Following the flight of STS-128, the tiles downstream of the protuberance were examined by TPS and material experts. Based on expert opinion and experience with the behavior of tile coating in arc jet facilities, it was determined none of the downstream tiles exceeded temperatures of $2500^{\circ} \mathrm{F}$. This information has proven useful in post-flight reconstruction activities. A close-up runway photograph of the protuberance is shown in Figure 18.

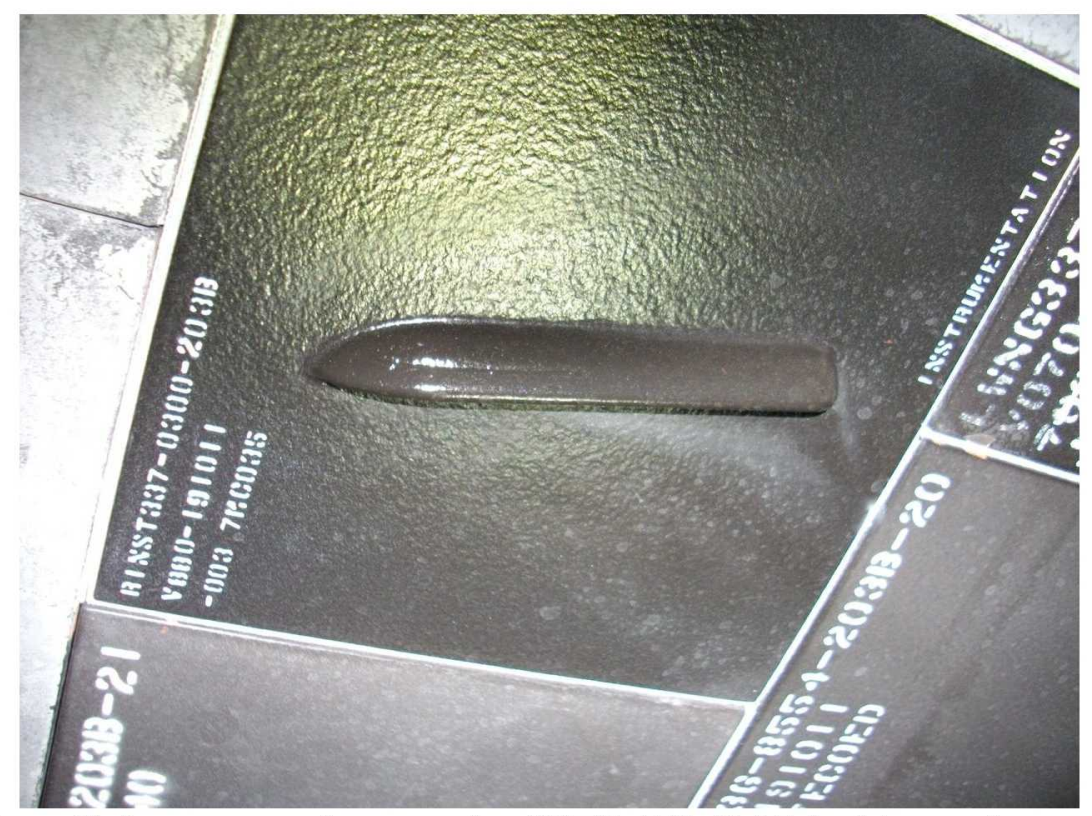

Figure 18. Post-flight runway photograph of STS-128 (0.35-inch) protuberance.

The protuberance tile was removed from the vehicle in a non-destructive manner for further evaluation and sent to material experts for additional study. The tile was scanned prior to the mission and will be scanned using an Optigo measuring device to assess the protuberance shape change. In addition, the two catalytic coated tiles were removed from 
the vehicle and have been replaced with new LI-900 tiles. The catalytic coating did exhibit some coating flaking and RCG coating imperfections on the aft portion of the coating footprint (see Figure 19).

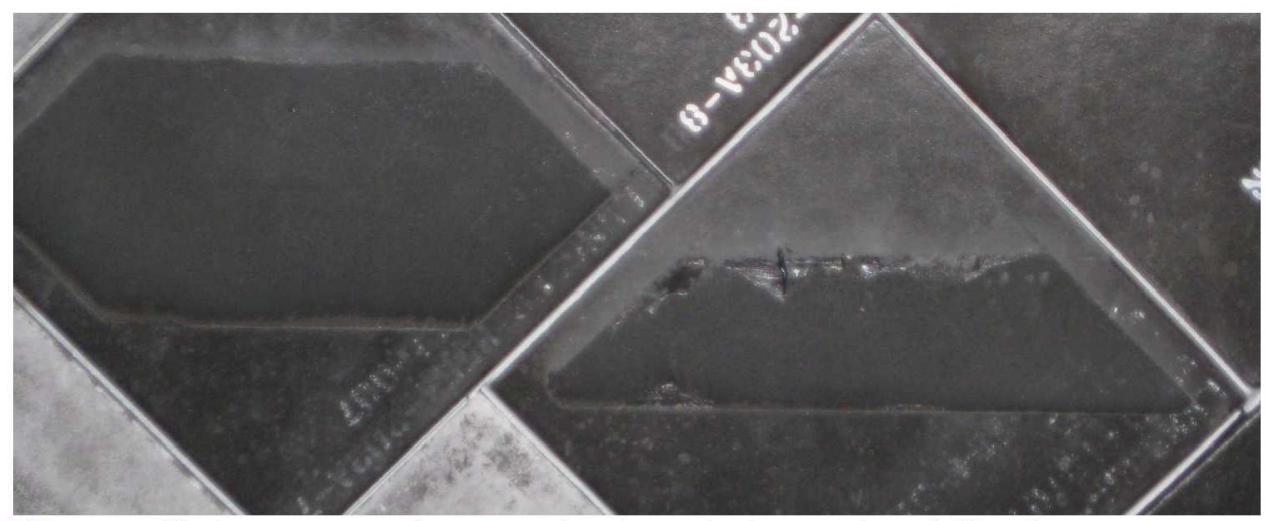

Figure 19. Post-flight runway photograph of catalytic coating following STS-128.

\section{Flight Data and Analysis Prediction Comparisons}

Comparisons between predicted and earliest observed BLT onset times were very favorable. As discussed in Ref (Berry), the predicted onset time for a 0.35-inch height using the BET was Mach 17.4. The observed BLT onset time at TC 1 was Mach 17.5. This is within the $\pm 1-\sigma$ prediction uncertainty of the BLT Tool (Ref. Berry).

Comparisons between predicted and observed temperatures for STS-128 were improved over the comparisons for STS-119. However, the analytical predictions were found to be higher than actual measurements. Of course, the ease of making comparisons is complicated by the upstream asymmetric BLT event. The available analysis results of most applicability to the flight data comparison are the results assuming an upstream BLT at Mach 16.2 (see Table 3). For that case, the predicted temperature on the protuberance was $2799^{\circ} \mathrm{F}$ (based on the pre-flight STS-128 trajectory) versus the maximum reported temperature on the protuberance of $2562^{\circ} \mathrm{F}$. Similar to STS -119 , the ability to compare predictions to actual flight data was less precise for the tiles surrounding the protuberance because of the sparseness of the instrumentation. However, material experts have stated that the tile coating temperature did not exceed $2500^{\circ} \mathrm{F}$. This is in contrast to the maximum pre-flight prediction of $2995^{\circ} \mathrm{F}$ for the protuberance tile acreage. However, it is believed that the temperatures were actually much lower than $2500^{\circ} \mathrm{F}$ and so the over-prediction is most much more severe than this initial comparison suggests. It is also important to note that the analysis summarized in Table 3 is for an early BLT event than that experienced on STS-128.

Due to the better placement of TC5, it is believed that future analysis predictions will be more accurate for the tile acreage surrounding the protuberance. However, in order to obtain ideal data to improve predictions in the peak vortex-heating region, an instrument would need to be placed immediately downstream of the protuberance.

\section{$\underline{\text { Future Missions }}$}


The BLT FE team is currently assessing the STS-119 and STS-128 data to understand all the implications of the data on potential future flights of the FE. Because of ground operations schedule constraints, a decision on the plans for the next flight of the experiment (currently planned for STS-131 in March 2010) was needed relatively soon following STS-128. A recommendation from the BLT FE team was accepted by the SSP to fly a 0.35 -in protuberance again on STS-131. While a strong desire within the team exists to test at an incrementally higher protuberance height, sufficient rationale for recommending the taller height could not be developed. Two observations prevented this rationale from being developed. The first was that the protuberance thermocouple produced questionable results that did not agree with our current understanding of physics. Thus, the ability of the team to show analytically (without adequate flight data for the 0.35 -in protuberance) that the Orbiter would be safe was tenuous. The second reason that rationale for the taller height could not be reached was the behavior of the other instrumentation, including the thermocouples installed in the catalytic-coated tiles. The steadily declining temperatures and drop-outs are not currently understood, although they have been seen on previous flights. It would provide invaluable data to the instrumentation community if the thermocouple behavior were repeated for the same protuberance and tile instrumentation configuration.

It should be noted that one minor change to the instrumentation was recommended and approved. For STS-131, only one tile will be coated with catalytic coating. That catalytic-coated tile will be instrumented with two thermocouples. One thermocouple will be at the leading edge of the coating region while the second thermocouple will be in the middle of the coating area.

A fourth flight of the BLT FE is also a possibility. The current SSP manifest calls for the final flight of the Program to be flow on Discovery (STS-133), which would presumably provide the opportunity for one additional flight of the flight experiment. The BLT FE team will make recommendations on what modifications should be made for STS-133 based on lessons learned from STS-131.

\section{Conclusions and Summary}

In support of the Boundary Layer Transition Flight Experiment (BLT FE) Project, a manufactured protuberance tile was installed on the port wing of Space Shuttle Orbiter Discovery for the flights of STS-119 and STS-128. Additional instrumentation was also installed in order to obtain more spatially resolved measurements downstream of the protuberance. This instrumentation measured boundary layer transition and associated temperatures at flight conditions. Comparisons of analytical predictions and the obtained flight data have shown that while BLT onset times have been accurately predicted using the engineering correlations, temperature predictions have large discrepancies with respect to the measured temperatures. The reason for these discrepancies is currently unknown. To obtain additional data to increase understanding of BLT, turbulent flow, and gas-surface interactions, two additional flights of the flight experiment are currently in the planning stages. 


\section{References}

Campbell, C.H., Anderson, B.P., King, R.A., Kegerise, M.A., Berry, S.A., Horvath, T.J., "Roles of Engineering Correlations in Hypersonic Entry Boundary Layer Transition Prediction," AIAA 2010-420.

Campbell, C.H., Garske, M.T., Kinder, G., Berry, S.A., “Orbiter Entry Boundary Layer Flight Testing", AIAA 2008-635.

CB-07-045, Letter from CB/Chief, Extravehicular Activity Branch, "Extravehicular Activity (EVA) Crew Consensus Report for EVA Removal of a Boundary Layer Transition (BLT) Detailed Test Objective (DTO) Fixed Protuberance", July 12, 2007.

Bertin, J.J., Schneider, S.P., Campbell, C.H., “A Flight-Testing Proposal on RoughnessInduced Boundary-Layer Transition on the Space Shuttle Orbiter", NASA White Paper, November 14, 2006.

Berry, S.A., King. R.A., Kegerise, M.A., Wood, W., McGinley, C., Anderson, B.P., "Updated to Orbiter Boundary Layer Transition Prediction Tool," AIAA 2010-246, January 2010.

Wright, M.J., Candler, G.V., and Bose, D., "Data-Parallel Line Relaxation Method for the Navier-Stokes Equations," AIAA Journal, Vol. 36, No. 9, pp. 1603-1609, Sept. 1998.

Campbell, C.H., et.al, “Orbiter Return to Flight Entry Aeroheating”, AIAA 2006-2917, June 2006.

Spanos, T., Kinder, G., Campbell, C.H., Andress, J., "Boundary Layer Transition Flight Experiment Thermal Protection System and Instrumentation Modifications", AIAA 2010-422, January 2010.

Berry, S.A., Horvath, T.J., Cassady, A.M., Kirk, B.S., Wang, K.C., Hyatt, A.J., "Boundary Layer Transition Results from STS-114", AIAA 2006-2922.

Marichalar, J.J., Rochelle, W.C., Kirk, B.S., Campbell, C.H., "BLIMPK/Streamline Surface Catalytic Heating Predictions on the Space Shuttle Orbiter", AIAA 2006-180.

McGinley, C.B., Berry, S.A., Kinder, G.R., Barnwell, M., Wang, K.C., Kirk, B.S., "Review of Orbiter Flight Boundary Layer Transition Data", AIAA 2006-2921.

Kirk, B.S., "wedge: A Tool for Predicting Turbulent Zones of Influence for Discrete Roughness Transition Events", NASA JSC EG-SS-06-02, 2006.

Tang, C., Wood, W.A., "Numerical Simulations of the Boundary Layer Transition Flight Experiment", AIAA 2010-XXXX. 
Horvath, T.J., Tomek, D.M., Berger, K.A., Zalameda, J.N., Splinter, S.C., Krasa, P.W., Schwartz, R.J., Gibson, D.M., Tietjen, A.B., Tack, S., "The HYTHIRM Project: Flight Thermography of the Space Shuttle during Hypersonic Flight", AIAA 2010-241.

Spisz, T. S., Taylor, J. C., Gibson, D. M., Horvath, T. J., Zalameda, J. N., Tomek, D. M., Tietjen, A. B., Tack, S., and Bush, B., "Processing Near-Infrared Imagery of Hypersonic Space Shuttle Reentries," SPIE Paper 7661-17, Apr. 2010, Thermosense XXXII Conference at the SPIE Symposium on Defense, Security, and Sensing. 